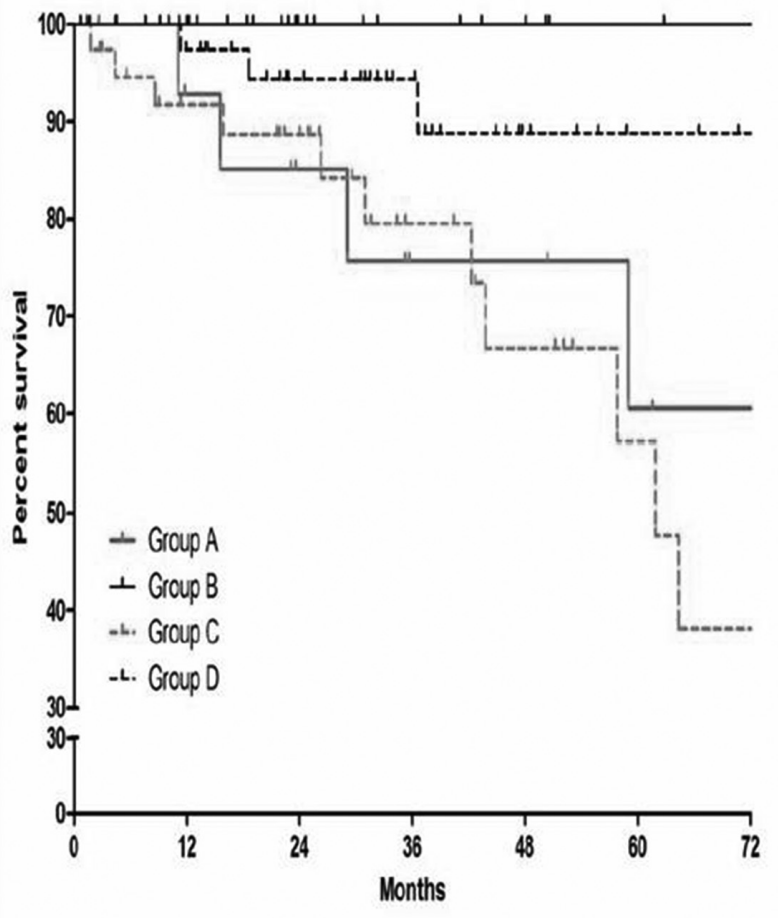

Notes: Group A=SLE-PAH without target therapy and responder; Group B=SLE-PAH without target therapy and nonresponder; Group C=SLE-PAH with target therapy and responder; Group D=SLE-PAH with target therapy and nonresponder;

Abstract 243 Figure 1 Survival of patients with SLE-PAH treated with intensiveimmunosuppressive therapy with or without PAH-target therapy

therapy on urinary angiostatin as a marker of activity in juvenile lupus.

Methods Fifty female patients with juvenile systemic lupus erythematosus (SLE) were enrolled in this study for twelve weeks trial of high dose of oral vitamin D weekly. We used radioimmunoassay to measure Serum levels of vitamin D. Systemic Lupus Erythematosus Disease Activity Index (SLEDAI) and the European Consensus Lupus Activity Measurement (ECLAM) were measured to assess lupus activity. Urinary angiostatin was evaluated as marker of activity. All parameters were measured on the day one of study and after three months. Thirty juvenile SLE patients as control.

Results At beginning of study all patients and control were almost similar regarding age, clinical, laboratory, urinary angiostatin and vitamin D levels. After three months the mean Vitamin D level was increase in patients group received Vitamin $D$ than in control group $(p<0.001)$. There was significant decrease in urinary angiostatin $(p<0.05)$ was observed in the patients received vitamin $\mathrm{D}$ compared to patient without vitamin D supplementation.

Conclusions Our findings show that high dose of vitamin D supplementation up to 12 weeks is safe and diminish lupus activity. We need longer duration and more studies to confirm our results.

\section{HIGH TITER ANA WITH ANTI-DFS70 ALONE IS NOT TO BE CONSIDERED A VALID CRITERION FOR LUPUS}

${ }^{1} E$ Chan*, ${ }^{2}$ L Andrade, ${ }^{3} J$ Damoiseaux, ${ }^{4} \mathrm{M}$ Satoh. ${ }^{1}$ USA; ${ }^{2}$ Universidade Federal de São Paulo, Rheumatology, Sao Paulo, Brazil; ${ }^{3}$ Maastricht University Medical Centre, Central Diagnostic Laboratory, Maastricht, The Netherlands; ${ }^{4}$ University of Occupational and Environmental Health, Clinical Nursing, Kitakyushu, Japan

\subsection{6/lupus-2017-000215.246}

Background and aims Positive ANA is one of Criteria for Classification of SLE for ACR and SLICC. As a follow-up to the International Consensus on ANA Patterns (ICAP) initiative (ANApatterns.org), the relevance of each ANA pattern is being re-evaluated.

Methods ANA test at 1/80 screening dilution was performed in 269 sequentially selected patients with SLE diagnosis, 918 healthy individuals, and 558 patients with non-SARD conditions. ANA interpretation was the consensus of 3 independent readers using 2 HEp-2 cell slide brands at 400x mag. Conversely, sequentially selected individuals presenting $>1 / 640$ titer Nuclear Dense Fine Speckled (DFS) ANA pattern (AC-2) in a large clinical laboratory within a 2 year period had the diagnosis assessed by interview with the respective physician.

Results Among 269 consecutive SLE patients, 96.3\% had a positive ANA with the following principal nuclear patterns: homogeneous (29.3\%), coarse speckled (14.7\%), fine speckled $(40.1 \%)$. Only one patient $(0.3 \%)$ had the DFS pattern and the reactivity to DFS70 confirmed by ELISA. Conversely, among 118 ANA+ healthy individuals and 102 ANA+ patients with miscellaneous non-SARD conditions, 33\% and $17 \%$ presented the DFS pattern, respectively. In addition, the 327 consecutive high-titer DFS individuals presented mostly non-SARD conditions or non-specific clinical presentation. Only 7 had possibly SARD-related presentations: 1 anti-phospholipid syndrome, 1 "possible" SLE (polyarthritis, arthritis, chronic urticaria), $1 \mathrm{WG}, 1 \mathrm{DLE}, 1 \mathrm{PBC}$, and 1 RA.

Conclusions Well-defined anti-DFS ANA, confirmed by antigen-specific reflex testing, should not be considered a criterion for SLE - either in the ACR or SLICC classification criteria.

\section{THE EFFICACY OF ANTI-CD20 ANTIBODY RITUXIMAB FOR REFRACTORY PATIENTS WITH SYSTEMIC LUPUS ERYTHEMATOSUS}

S Imata*, K Saito, N Ohkubo, S Nakayamada, K Nakano, S Kubo, M Yoshikawa, Y Miyazaki, H Yoshinari, Y Tanaka. University of Occupational and Environmental Health, The First Depertment of Internal Medicine, Kitakyushu, Japan

\subsection{6/lupus-2017-000215.247}

Background and aims B cells play a crucial role in pathogenesis of Systemic Lupus Erythematosus(SLE). We examined the efficacy of B cell depletion therapy rituximab for refractory patients with SLE.

Methods 63 eligible study subjects since 2002 until 2015 were men and women, who met the American College of Rheumatology criteria in 1987 or SLICC2012 for the classification of SLE. The protocols were approved by the Institutional Review Board of our university. Treatment protocol: 2 or 4 weekly doses of $500 \mathrm{mg} /$ body, 2 biweekly doses of $1000 \mathrm{mg} /$ body or 4 weekly doses of $1000 \mathrm{mg} /$ body.

Results Baseline characteristics; gender $\mathrm{M}: \mathrm{F}=6: 57$, age 33.9 years, disease duration 87.2 months, organ failure NPSLE:35, lupus nephritis:46, treated with IVCY 34/63. The 60/63 
Abstract 244 Table 1 Independent predictors of morality obtained by univariate analysis

\begin{tabular}{|c|c|c|c|}
\hline Univariate analysis & HR & $95 \%$ Cl & $p$ \\
\hline Age at onset $\geq 50$ years & 3.935 & $2.111-7.334$ & $<0.001$ \\
\hline Onset to diagnosis \1 year & 1.996 & $1.251-3.186$ & 0.004 \\
\hline Gender & 2.082 & $1.1199-3.873$ & 0.021 \\
\hline Baseline organ damage* & 2.846 & $1.757-4.610$ & $<0.001$ \\
\hline Renal involvement* & 2.434 & $1.425-4.156$ & 0.001 \\
\hline Hematologic involvement* & 1.605 & $0.978-2.635$ & 0.061 \\
\hline Interstitial lung disease* & 2.167 & $0.973-4.823$ & 0.058 \\
\hline Pulmonary arterial hypertension* & 4.126 & 2.107-8.081 & $<0.001$ \\
\hline Neuropsychiatric involvement* & 2.290 & $1.169-4.486$ & 0.016 \\
\hline Serositis* & 2.580 & $1.605-4.148$ & $<0.001$ \\
\hline No. of involved organ systems (1) * & 1.793 & $0.719-4.466$ & 0.210 \\
\hline No. of involved organ systems (2)* & 2.045 & $0.805-5.195$ & 0.133 \\
\hline №. of involved organ systems $(\geq 3)^{*}$ & 5.638 & 2.339-13.589 & $<0.001$ \\
\hline
\end{tabular}

* Performed after controlling of gender, onset age and time from onset to diagnosis

Abstract 244 Table 2 Independent predictors of morality obtained by multivariate analysis

\begin{tabular}{llll}
\hline Multivariate analysis & HR & $95 \% \mathrm{Cl}$ & $p$ \\
\hline Age at onset $\geq 50$ years & 3.311 & $1.775-6.176$ & $<0.001$ \\
Onset to diagnosis $\geq 1$ year & 1.645 & $1.019-2.656$ & 0.042 \\
Baseline organ damage & 2.292 & $1.400-3.755$ & 0.001 \\
No. of involved organ systems (23) & 4.669 & $1.919-11.362$ & 0.001 \\
\hline
\end{tabular}

patients were trailable at 1 year. Primary endpoint; disease activity scores were significantly improved (SLEDAI $17.3 \rightarrow 3.0$, BAILAG $17.8 \rightarrow 2.2$; LOCF). The achievement ratio of major clinical response (MCR) defined as no BILAG A and/or B score was $61.7 \%$. Secondary endpoint; dose of corticosteroid was decreased from 43.2 to $8.4 \mathrm{mg} /$ day. Nephritis score originally designed (total 10 points; protein 3 , occult blood 3, cast 4) was decreased (3(before) $\rightarrow 2.3$ (1month) $\rightarrow 0.8$ (1year)). The $37 / 50$ patients were trailable at 5 years. Primary failure: 3 (8\%), secondary failure (flare) : 12 (32.8\%, duration: 23.8 months), MCR: 18 (48.6\%), corticosteroid discontinued: 5 among 37 patients.

Conclusions Rituximab showed short-term and long-term efficacy in refractory patients with SLE, although some of patients had a relapse. Rituximab could be considered as a therapeutic strategy for refractory patients with SLE. 